\title{
Most Cited Articles in 2020
}

Dear Readers

We would like to announce the top 11 articles most frequently cited in the 2018 and 2019 issues of the International Heart Journal. The numbers of citations are cited in the year of 2020.

\begin{tabular}{|c|c|c|c|}
\hline Rank & Authors/Title/Issue & Type & $\begin{array}{l}\text { Number } \\
\text { of citation }\end{array}$ \\
\hline 1 & $\begin{array}{l}\text { Yang Bai, Qiangnu Zhang, Yijiang Su, Zhenye Pu, Kunsheng Li. Modulation of the } \\
\text { Proliferation/Apoptosis Balance of Vascular Smooth Muscle Cells in } \\
\text { Atherosclerosis by lncRNA-MEG3 via Regulation of miR-26a/Smad1 Axis. 2019; } \\
60(2): 444-450 .\end{array}$ & $\begin{array}{c}\text { Experimental } \\
\text { Study }\end{array}$ & 12 \\
\hline 2 & $\begin{array}{l}\text { Xiaoping Lin, Sichen Zhang, Zhaoxia Huo. Serum Circulating miR-150 is a } \\
\text { Predictor of Post-Acute Myocardial Infarction Heart Failure. 2019; 60(2): 280-286. }\end{array}$ & Clinical Study & 11 \\
\hline 2 & $\begin{array}{l}\text { Zhi-Dong Ge, Qingquan Lian, Xiaowen Mao, Zhengyuan Xia. Current Status and } \\
\text { Challenges of NRF2 as a Potential Therapeutic Target for Diabetic } \\
\text { Cardiomyopathy. } 2019 ; 60(3): 512-520 .\end{array}$ & Review & 11 \\
\hline 4 & $\begin{array}{l}\text { Isao Nishi, Yoshihiro Seo, Yoshie Hamada-Harimura, Kimi Sato, Seika Sai, } \\
\text { Masayoshi Yamamoto, Tomoko Ishizu, Akinori Sugano, Kenichi Obara, Longmei } \\
\text { Wu, Shoji Suzuki, Akira Koike, Kazutaka Aonuma, Ibaraki Cardiovascular } \\
\text { Assessment Study-Heart Failure Investigators. Utility of Nutritional Screening in } \\
\text { Predicting Short-Term Prognosis of Heart Failure Patients. 2018; 59(2): 354-360. }\end{array}$ & Clinical Study & 9 \\
\hline 4 & $\begin{array}{l}\text { Shouwen Zhang, Rongjun Zhang, Fangfang Wu, Xinhua Li. MicroRNA-208a } \\
\text { Regulates H9c2 Cells Simulated Ischemia-Reperfusion Myocardial Injury via } \\
\text { Targeting CHD9 through Notch/NF-kappa B Signal Pathways. 2018; 59(3): 580- } \\
588 .\end{array}$ & $\begin{array}{l}\text { Experimental } \\
\text { Study }\end{array}$ & 9 \\
\hline 4 & $\begin{array}{l}\text { Yasuo Okumura, Katsuaki Yokoyama, Naoya Matsumoto, Eizo Tachibana, } \\
\text { Keiichiro Kuronuma, Koji Oiwa, Michiaki Matsumoto, Toshiaki Kojima, Ken } \\
\text { Arima, Tomobumi Kotani, Kazumiki Nomoto, Yukitoshi Ikeya, Seiji Fukushima, } \\
\text { Motoyuki Onikura, Yasuko Suzuki, Masami Fujita, Hideyuki Ando, Norikazu } \\
\text { Ishikawa, Atsushi Hirayama, for the SAKURA AF Registry Investigators. Patient } \\
\text { Satisfaction with Direct Oral Anticoagulants and Warfarin: Findings from the } \\
\text { SAKURA AF Registry. 2018; 59(6): 1266-1274. }\end{array}$ & Clinical Study & 9 \\
\hline 7 & $\begin{array}{l}\text { Chunyang Tian, Jia Song, Dongxu He, Jiake Wu, Zhijun Sun, Zhaoqing Sun. } \\
\text { Predictive Value of Mean Platelet Volume/Platelet Count for Prognosis in Acute } \\
\text { Myocardial Infarction. 2018; 59(2): 286-292. }\end{array}$ & Clinical Study & 8 \\
\hline 7 & $\begin{array}{l}\text { Chaofeng Miao, Haixia Cao, Yonggan Zhang, Xueli Guo, Zifan Wang, Jiaxiang } \\
\text { Wang. LncRNA DIGIT Accelerates Tube Formation of Vascular Endothelial Cells } \\
\text { by Sponging miR-134. 2018; 59(5): 1086-1095. }\end{array}$ & $\begin{array}{l}\text { Experimental } \\
\text { Study }\end{array}$ & 8 \\
\hline 7 & $\begin{array}{l}\text { Yang Yu, Ming Zhang, Yali Hu, Yali Zhao, Fei Teng, Xiaoyan Lv, Ji Li, Ying } \\
\text { Zhang, Grant M. Hatch, Li Chen. Increased Bioavailable Berberine Protects } \\
\text { Against Myocardial Ischemia Reperfusion Injury Through Attenuation of NF } \\
\text { kappa B and JNK Signaling Pathways. 2018; 59(6): 1378-1388. }\end{array}$ & $\begin{array}{c}\text { Experimental } \\
\text { Study }\end{array}$ & 8 \\
\hline 7 & $\begin{array}{l}\text { Takatoyo Kiko, Akiomi Yoshihisa, Yuki Kanno, Tetsuro Yokokawa, Satoshi Abe, } \\
\text { Makiko Miyata-Tatsumi, Tomofumi Misaka, Masayoshi Oikawa, Atsushi } \\
\text { Kobayashi, Takafumi Ishida, Yasuchika Takeishi. A Multiple Biomarker Approach } \\
\text { in Patients with Cardiac Sarcoidosis. 2018; 59(5): 996-1001. }\end{array}$ & Clinical Study & 8 \\
\hline 7 & $\begin{array}{l}\text { Yusuke Watanabe, Kenichi Sakakura, Yousuke Taniguchi, Kei Yamamoto, Hiroshi } \\
\text { Wada, Shin-ichi Momomura, Hideo Fujita. Determinants of Slow Flow in } \\
\text { Percutaneous Coronary Intervention to the Culprit Lesion of Non-ST Elevation } \\
\text { Myocardial Infarction. 2018; 59(6): 1237-1245. }\end{array}$ & Clinical Study & 8 \\
\hline
\end{tabular}

We appreciate all authors who submitted their manuscripts to the International Heart Journal, and look forward to receiving manuscripts with high scientific impact from all over the world.

Issei Komuro, MD

Editor-in-Chief

International Heart Journal

November 2021 